\title{
A Qualitative Exploration of Perceptions of a Digital Intervention to Promote Physical Activity in Older Adults
}

\author{
Sebastien Pollet, James Denison-Day, Katherine Bradbury, Rosie Essery, Elisabeth Grey, \\ Max Western, Fiona Mowbray, Kirsten A. Smith, Joanna Slodkowska-Barabasz, Nanette Mutrie, \\ Paul Little, and Lucy Yardley
}

\begin{abstract}
Purpose: This study explored participant views of a web-based physical activity intervention for older adults and examined how they resonate with the key principles that guided intervention development. Methods: Qualitative interviews were carried out with 52 older adults. A deductive qualitative analysis approach was taken, based around the intervention's key principles. Results: Participants expressed mostly positive views of the intervention features, broadly confirming the appropriateness of the key principles, which were to: (a) encourage intrinsic motivation for physical activity, (b) minimize the risk of users receiving activity suggestions that are inappropriate or unsafe, (c) offer users choice regarding the activities they engage with and build confidence to undertake more activity, and (d) minimize the cognitive load and need to engage with the intervention website. The findings also identified ways in which content could be improved to further increase acceptability. Conclusion: This study illustrates how using the person-based approach has enabled the identification and implementation of features that older adults appreciate.
\end{abstract}

Keywords: acceptability, behavior change, digital physical activity intervention

Evidence shows that an active lifestyle has a range of benefits for cognitive, physical, and psychological health, including improved cardiovascular and respiratory health; enhanced insulin sensitivity; heightened bone and muscle strength; improved positive affect and cognitive function; and increased resistance to Type 2 diabetes, cancers, and depression (Corder, Ogilvie, \& van Sluijs, 2009; Kohl et al., 2012; Powell, Paluch, \& Blair, 2011). The UK Chief Medical Officers' Physical Activity Guidelines advise that older adults engage in at least 150 min of moderate-intensity aerobic activity per week, perform strength and balance exercises twice a week, and reduce the amount of sedentary time (Department of Health and Social Care, 2019).

While many interventions have been developed to increase rates of physical activity in older adults, evidence suggests there has been little increase in physical activity levels of the global older adult population (Antonucci et al., 2012; Clarke, Norris, \& Schiller, 2017; Kohl et al., 2012; Lachman, Lipsitz, Lubben, Castaneda-

(C) 2021 The Authors. Published by Human Kinetics, Inc. This is an Open Access article distributed under the terms of the Creative Commons Attribution 4.0 International License, CC BY 4.0, which permits unrestricted noncommercial and commercial use, distribution, and reproduction in any medium, provided the original work is properly cited, the new use includes a link to the license, and any changes are indicated. See http://creativecommons.org/licenses/by/4.0. This license does not cover any third-party material that may appear with permission in the article.

Pollet and Denison-Day are joint first authors as they have contributed equally. Pollet, Denison-Day, Bradbury, Essery, Mowbray, Smith, Slodkowska-Barabasz, and Yardley are with the Centre for Clinical and Community Applications of Health Psychology, University of Southampton, Southampton, Hampshire, United Kingdom. Grey and Western are with the Department for Health, University of Bath, Bath, Somerset, United Kingdom. Mutrie is with the Physical Activity for Health Research Centre, The University of Edinburgh, Edinburgh, United Kingdom. Little is with the Primary Care, Population Sciences and Medical Education, University of Southampton, Southampton, Hampshire, United Kingdom. Yardley is with the School of Psychological Science, University of Bristol, Bristol, United Kingdom. Pollet (sebastien.pollet@soton.ac.uk) is corresponding author.
Sceppa, \& Jette, 2018). Indeed, in the United Kingdom, current estimates suggest that $35 \%$ of older adults aged $65-74$ years, $52 \%$ aged $75-84$ years, and $74 \%$ aged 85 years or above do not meet the aerobic recommendations. Moreover, only $16 \%$ of older adults aged $65-74$ years and $8 \%$ aged 75 years or above are thought to meet the strength recommendations (Health and Social Care Information Centre, 2017). Many physical activity interventions require users to follow a formal exercise regimen, often setting aside time to go to a gym or use specialist equipment (Lachman et al., 2018), or expect daily engagement with the intervention. Such interventions are often difficult to implement on a sufficient scale to influence changes in physical activity at national levels (Koorts et al., 2018). A potentially more effective alternate approach, involving encouraging users to integrate physical activity into their daily routines based on personalized goals and interests, has been suggested by the National Institute on Aging Go4Life Program (National Institute on Aging, 2019).

A potential way to overcome the problem of scaling is to utilize digital behavior change interventions. These are remotely delivered using technologies, such as websites and mobile applications (Stockwell et al., 2019). Using these technologies allows for the creation of "persuasive systems" (Oinas-Kukkonen \& Harjumaa, 2008), which utilize features of the technology to support behavior change via various methods, such as primary task support (e.g., tailoring and self-monitoring), dialog support (e.g., praise and reminders), system credibility support (e.g., trustworthiness and expertise), and social support (e.g., social facilitation and cooperation) (Oinas-Kukkonen \& Harjumaa, 2009). Further to this, delivering interventions via the Internet overcomes restrictions introduced by the physical location of both the user and intervention resources, overcoming geographic boundaries and enabling widespread, costeffective dissemination (Bennett \& Glasgow, 2009). In the United Kingdom, older adults are the fastest growing group of Internet users: Internet usage in those aged 65-74 years rose from 52\% in 2011 to $83 \%$ in 2019 ; in those aged $75+$ years, usage rose from $20 \%$ in 2011 to 47\% in 2019 (Office for National Statistics, 2019). While 
it must be acknowledged that digital behavior change interventions are not suitable for everyone, such as those who may not have access to the Internet, by using this approach to deliver effective interventions at scale very cheaply, existing resources can be freed up to reach those without digital access in other ways (e.g., through faceto-face programs).

Indeed, using digital approaches to deliver interventions has been demonstrated as an effective way to promote increased physical activity (Kohl, Crutzen, \& de Vries, 2013). There are numerous commercial websites and apps available to the general population, but these are often focused on younger, physically active adults with higher levels of technological literacy and often depend on smartphone technology (Davies, Spence, Vandelanotte, Caperchione, \& Mummery, 2012). However, there is also increasing evidence that digital physical activity interventions can be acceptable and effective among an older population (Ammann, Vandelanotte, de Vries, \& Mummery, 2013; Muellmann et al., 2016; Stockwell et al., 2019). Although such web-based interventions have been demonstrated to provide short-term improvements in physical activity, there is limited evidence as to their longterm benefits (Muellmann et al., 2016; Stockwell et al., 2019). Furthermore, there remains an uncertainty as to which intervention features are most likely to be acceptable and effective in an older adult population (Zubala et al., 2017), with calls for future research to identify what is acceptable and unacceptable to older adults about interventions to increase physical activity (French, Olander, Chisholm, \& Mc Sharry, 2014). This research therefore sought to understand in detail which aspects of a digital behavior change intervention to promote physical activity (Active Lives) were likely to be acceptable to older adults. In addition, this allowed for the identification of any ways in which these might need to be adapted as part of the iterative development process (O'Cathain et al., 2019). An intervention being acceptable to the intended user population is a key factor in intervention engagement (Corbett, Singh, et al., 2018; Madkins, Moskowitz, Moran, Dellucci, \& Mustanski, 2019; Sekhon, Cartwright, \& Francis, 2017), and therefore potential effectiveness, in terms of an intervention's targeted behavior changes when used over a longer period of time.

"Active Lives," a novel web-based intervention, was developed with the aim of promoting long-term increases in physical activity and, by extension, delaying or preventing cognitive decline (Buchman et al., 2012; Norton, Matthews, Barnes, Yaffe, \& Brayne, 2014), in older adults with and without existing age-related cognitive decline. The Active Lives intervention was developed using an evidence-, theory-, and person-based approach (Morrison, Muller, Yardley, \& Bradbury, 2018; Yardley, Morrison, Bradbury, \& Muller, 2015), which uses in-depth qualitative research in an iterative process to obtain an understanding of user views, context, and experiences. This is then used to adapt and optimize the intervention to ensure it is maximally meaningful, feasible, and engaging for the target population. As part of this iterative development process (Moore et al., 2015), qualitative research was conducted to explore user perceptions and experiences of the intervention, whose provisional content was underpinned by the theory and evidence-based "guiding principles," which aim to briefly summarize the key intervention features designed to promote behavior change (Yardley, Morrison, et al., 2015). Understandings of users' preferences and experiences helped to refine the intervention's underpinning guiding principles and identified which aspects of the intervention older adults found either acceptable or unacceptable.

Using a combination of think-aloud (Charters, 2003) and semistructured retrospective interviews (Fylan, 2005), this work sought to explore older adults' perceptions of the key intervention features of Active Lives in order to understand more about whether they were implemented in a way that would be acceptable to users. In this context, we considered acceptable to mean that the content and design of the intervention (e.g., the overall message, format, functionality, navigation and wording) is easily understandable, engaging (in that users are inclined to continue using it), and persuades the user to engage in the suggested behaviors. This approach presented an opportunity to understand how the proposed mechanisms through which the intervention was expected to work were viewed by prospective users and, therefore, to identify ways in which the intervention could be improved specifically for the target population as part of an iterative design process to increase the likelihood of effective behavior change (O'Cathain et al., 2019). This work was conducted as part of the intervention development phase of a larger body of research into the "Active Brains" intervention, of which Active Lives forms a key component. Active Brains aims to prevent and reduce cognitive decline in older adults through increased physical activity (Active Lives), online brain training, and healthy eating.

\section{Methods}

\section{Design}

After ethical approval for the study was obtained from the South Central-Hampshire B Research Ethics Committee, reference number 17/SC/0463, data collection was carried out in two phases. First, think-aloud interviews were conducted on preliminary intervention materials, which informed the iterative development of intervention content and the completion of a full prototype of the website. Following this, a qualitative feasibility evaluation of the intervention was conducted, which involved participants having access to the intervention for 3 weeks and then providing feedback in semistructured interviews. This formed a brief part of the development phase study intended to collect perceptions and views on different aspects of the intervention. Each of these phases was intended to provide complementary data regarding participants' experiences of using the intervention in order to provide a potentially broader insight into their perceptions of it. Think-aloud interviews generate immediate reactions to the content but require the presence of a researcher, which may influence responses. In contrast, retrospective interviews allow participants to use the intervention in their own environment, which can highlight additional issues with the intervention (such as with navigation and usability) as well as barriers to behavior, which cannot be identified during think-aloud interviews.

\section{Participants}

Fifty-two participants were recruited from two routes: invitation through Join Dementia Research (a public facing web portal for matching U.K.-based patients and public to relevant studies) and searches of general practice databases.

To be eligible for inclusion, participants had to meet the following criteria:

- Be retired and aged between 60 and 85 years old

- Not in receipt of terminal/palliative care

- Not having severe mental health problems (including an existing diagnosis of dementia) and/or major uncontrolled depression/schizophrenia 
- Not already meeting Department of Health and Social Care's weekly physical activity recommendations (150 min of moderate exercise, or 75 min of vigorous exercise)

Eligible participants provided informed consent by completing an online consent form and answered some brief online eligibility screening questionnaires. The screening questionnaires comprised the Baddeley Grammatical reasoning task (Baddeley, 1968) and the International Physical Activity Questionnaire Short (Craig et al., 2003). Eligible participants were assigned to one of two groups based on the results of cognitive assessments: participants scoring $1 S D$ below the norm for the Baddeley reasoning test task (Baddeley, 1968) were assigned to the lower cognitive score group, while participants who scored above this criterion were assigned to the higher cognitive score group. Cognitive assessment scores were collected due to this study being part of a wider body of research for which Active Lives was developed. In this context, we were interested in finding out if Active Lives is suitable for people with mild cognitive impairment, and whether their views of the intervention differ.

\section{Guiding Principles}

A key part of the person-based approach is the development of guiding principles, which specify the intervention's core design objectives (what the intervention is trying to achieve) and the key intervention features that will support achievement of those design objectives within the user context. The design objectives specify what the intervention must do in order to address the needs of the target user and enhance engagement with the intervention. The guiding principles offer a succinct summary of the crucial ways in which the intervention is intended to support behavior change by improving engagement with the intervention content.

To draft provisional guiding principles, an understanding of target users was obtained from scoping reviews and the research team, including Patient and Public Involvement members, and combined with previous research evidence and the application of theory. The development of Active Lives drew on three key theories; self-determination theory (Deci \& Ryan, 1985; Ryan \& Deci, 2000), social cognitive theory (Bandura, 1986, 1991), and habit formation (Aarts, Paulussen, \& Schaalma, 1997; Lally \& Gardner, 2013).

- Self-determination theory proposes that more self-determined/ higher quality motivation and continued engagement in a behavior is underpinned by conditions that support the three basic psychological needs: the need for autonomy, the need for competence, and the need for relatedness.

- Social cognitive theory presents a three-way model in which personal factors, including sense of agency, self-regulation and self-efficacy, environmental influences, and behavior, continually interact. This theory also highlights the importance of outcome expectations, which are subjective estimates of how likely a behavior will result in a particular outcome.

- Habit formation relates to the idea that physical activity behaviors are capable of being automatically evoked without the need of significant intentional effort or extensive planning when incorporated in existing lifestyles.

The resulting guiding principles (Table 1) identified four key design objectives needed to ensure that Active Lives would meet older adults' behavioral needs in order for them to successfully increase physical activity:

(a) Older adults may not be sufficiently motivated to increase activity. To build intrinsic motivation, Active Lives therefore presents novel content that is appealing to older adults, such as strength and balance training, breaks from sitting (to reduce sedentariness), and activities that easily fit within older adults' lifestyles. The rationale for increasing activity draws on benefits that older adults have reported as salient and motivating, such as enjoyment, social interaction, maintenance of independence, and improvement in symptoms (Devereux-Fitzgerald, Powell, Dewhurst, \& French, 2016).

(b) The need to cater for a heterogeneous population of older adults with varying capabilities/preferences. Therefore, Active Lives aims to minimize the risk of users receiving activity suggestions that are too advanced/unsafe or too basic to increase capacity. A choice of activities, designed to be carried out independently and suit different abilities/ preferences, are presented. Content is tailored to participants' reported capability and preferences, to ensure it is beneficial and safe (e.g., in the strength and balance section, videos of simple chair-based exercises are presented to those who report problems with strength/balance; these slowly increase in intensity, working up to standing exercises over time). Participants choose the section of Active Lives that they would like to begin with (guidance steers people toward what is most beneficial/safe); as activity levels and confidence increase, the intervention encourages use of all sections.

(c) Many older adults have concerns about increasing activity (Devereux-Fitzgerald et al., 2016), Active Lives therefore aims to build confidence to undertake more activity by addressing common concerns, providing reassurance, encouragement, and techniques for overcoming barriers. Graded activity employs very gradual increases from participants' baseline. Participant stories model how similar individuals overcame concerns/ barriers.

(d) As nearly $20 \%$ of older adults will have some age-associated cognitive decline/mild cognitive impairment (Petersen, 2016), Active Lives aims to minimize cognitive load and dependence on the intervention website to ensure behavior can be maintained. Techniques used include examples of how to create new physical activity habits, including making small changes that fit within everyday routines; support with setting up prompts to behavior in the physical/social environment; and signposting to offline resources, such as local activity classes. Goal setting and reviewing (with tailored feedback to support motivation), along with the use of step counters, facilitate self-monitoring and self-regulation. E-mails provide additional prompts to behavior and contain behavior change techniques designed to sustain motivation for being active.

These guiding principles were used alongside the theoretical and empirical understandings of physical activity behavior in this population to decide on:

(a) appropriate features and functions of the intervention likely to increase in physical activity behaviors and

(b) the most appropriate behavior change techniques to deliver these functions, such as goal setting, action planning, restructuring the physical and social environment, instruction on how to perform a behavior, and habit formation (Michie et al., 2013).

Using this approach allows for the development of interventions that are more likely to be acceptable to the target population (Yardley, Ainsworth, Arden-Close, \& Muller, 2015) and, as such, increases the likelihood of effectiveness. 


\section{User context \\ In older people: \\ (a) Levels of physical activity are low and often unchanged by interventions \\ (b) Improving health is not usually a strong motivation for physical activity}

Catering for highly heterogeneous population in terms of physical activity capabilities and preferences

Many older people have doubts and concerns about the safety of activity and their capability to undertake activity

\section{Key design objective}

Encourage engagement with and intrinsic motivation for physical activity

- Minimize risk of users receiving activity suggestions that are too advanced and unsafe or too basic and those that do not increase capacity

- Steer users toward those activities that seem most personally suitable/ beneficial

- Offer users choice and autonomy regarding content and activities they engage with (within those suitable)

Build confidence to undertake more activity

Provide reassurance, encouragement, techniques, and strategies to:

Reduce concerns and barriers:

- Common concerns pages specific to each section

- Provision of carefully graded activities with very gradual increases from low activity baseline

- Stories from similar others about their successes with building up activities

- Access to a trained supporter to support engagement

Increase self-efficacy:

- Graded goal setting and review

(a) Target population has some cognitive impairment

(b) Behavior must be maintained for 5 years

\author{
Minimize: \\ - Cognitive load \\ - Dependence on the \\ intervention website
}

- Suggestions/examples of how to create new physical activity habits

- Suggestions/examples of how to make small changes to everyday (physical and social) environment to prompt activities

- Support for planning environmental prompting for each activity goal set (time of day and links to specific activities)

- Exercise instructions/goals set/record sheets available as printouts

- Signposting/links to offline resources to support activity (e.g., activity classes in local area)

\section{Evidence base}

- Focusing on immediate benefits encourages users to engage in physical activity by building positive outcome expectations (Bandura, 1991; Devereux-Fitzgerald, Powell, Dewhurst, \& French, 2016; Notthoff, Klomp, Doerwald, \& Scheibe, 2016).

- Increases intrinsic motivation by encouraging engaging in physical activity for the feelings of fun, pleasure, and satisfaction as proposed in SDT (Deci \& Ryan, 1985; Ryan \& Deci, 2000).

- Offering activities and suggestions, such as use of pedometers (Bravata et al., 2007), that may be novel to users makes the intervention more engaging (Morrison, Yardley, Powell, \& Michie, 2012).

- Tailoring activity recommendations to selfreported capabilities reduces the risk of making suggestions that may be harmful to users.

- Allowing self-tailoring of information and goals from a range of options increases the likelihood that users will be able to select personally appropriate advice (Lorig \& Holman, 2003) as well as builds selfefficacy and supports the need for autonomy (Ryan \& Deci, 2000).

- Addressing common concerns provides users with an increased sense of competence in their ability to engage in physical activity (Deci \& Ryan, 1985; Ryan \& Deci, 2000).

- Presenting stories from individuals with whom users can relate creates the opportunity for observed positive reinforcement of the impacts of physical activity and builds a sense of relatedness (Bandura, 1977; Deci \& Ryan, 1985).

- Providing access to brief human support in conjunction with web-based interventions has been shown to improve engagement and outcomes (Dennison et al., 2014).

- Allowing users to set achievable goals and build up physical activity through graded activities; helps to build self-efficacy (Bandura, 2012; Ryan \& Deci, 2000).

- Changing the physical or social context in which a behavior takes place, also known as environmental restructuring, helps to prompt automatic changes to that behavior (Michie, Atkins, \& West, 2014).

- This process facilitates the formation of these behaviors as habits, eventually removing the need for ongoing planning and support (Aarts et al., 1997).

- Habits are automatic behavioral responses to environmental cues. When forming habits, individuals find that, with repetition, the cognitive effort required to act decreases, and initiation becomes "second nature" (Lally \& Gardner, 2013).

Note. SDT = self-determination theory. 


\section{Active Lives}

Active Lives contains three submodules that users are directed to differentially, depending on which is deemed to be most beneficial for them. While tailored recommendations are made about which submodule the users may find the most helpful, participants have access to all three submodules at any point.

- The "Getting Active" submodule provides support and ideas about activities to try to increase individuals' overall levels of lifestyle physical activity (e.g., doing more walking, activities at home, and involving friends and family).

- The "Strength and Balance Training" submodule provides video demonstrations of simple strength and balance exercises and suggestions about how these can be built into daily activities and offers the opportunity to create an exercise plan. The level of exercise difficulty is tailored to the user and their progress.

- The "Breaks from Sitting" submodule supports individuals in reducing sedentary behavior by suggesting simple changes that can be made to daily routines.

Active Lives was designed to promote both initial engagement with, and long-term adherence to, new physical activities. It aimed to do this by first introducing users to new activity suggestions and encouraging goal setting and self-monitoring and, later, by offering ideas for users to build these into daily routines, to promote habit formation over the longer term. Active Lives was designed to support those with limited time and resources, as well as those with lower literacy skills. Content was written in easily accessible language and focused on simple ways of increasing physical activity that can be readily integrated into daily routines, without the purchase of additional equipment (to minimize costs, users are offered a free step counter as part of the intervention). A logic model for the intervention has been included (see Supplementary Material 1 [available online]), as well as an overview of the intervention content (see Supplementary Material 2 [available online]). A detailed account of the intervention development is available elsewhere (Essery et al., 2020).

\section{Procedure}

The procedure for each phase is outlined later.

Think-aloud interviews. The initial phase of interviews included the think-aloud interviews, which took place face-to-face at either participants' homes or in a private room at the University of Southampton. The purpose of these interviews was to elicit users' views on preliminary intervention materials and directly observe their use of, and interaction with, them to inform any necessary modifications. Participants were asked to work through a prototype of the Active Lives website with a researcher sitting beside them at the computer, while speaking their thoughts on content, structure, and presentation out loud in real time.

Retrospective interviews. Retrospective interviews were conducted following the completion of the initial think-aloud interviews. Participants were invited to use the prototype intervention at home for 3 weeks, keeping a diary of their experiences. These diaries were not included within the analyzed data; participants were instead encouraged to use them to prompt a recall of their experiences during the interview. Semistructured interviews were conducted after this time, either face to face or via telephone, in order to understand users' experiences of the intervention and recommended activities, and to identify further ways in which the intervention could be improved. The interview schedule was informed by the preceding phase of work, with a focus on participants' general views of the website and the activities it recommended.

All participants were offered a $£ 10$ high street voucher before each phase of the research they participated in.

\section{Analysis}

Interview recordings were transcribed verbatim, added to QSR International's NVivo 12 qualitative data analysis software, and analyzed using thematic analysis (Braun \& Clarke, 2006). As the purpose of this research was not to construct a new theory, but rather to establish whether participant experiences of using the Active Lives program matched those predicted by the guiding principles, a deductive qualitative analysis approach (Crabtree \& Miller, 1999; Gilgun, 2013) was taken. This was conducted in six stages:

(a) The first two authors read the transcripts several times in order to familiarize themselves with the data and produced an a priori template of codes (Crabtree \& Miller, 1999) in the form of an initial coding manual based around the guiding principles for the Active Lives intervention.

(b) This coding manual was used to guide the identification of relevant extracts from both phases of data collection. Through this phase, the coding manual was continually updated and refined through discussion between the researchers, with a broad "other" code used for additional open coding to allow for the identification of experiences and understandings that could not be explained by the intervention's guiding principles.

(c) Following this, the collated codes were explored to determine how well they fit within the deductive framework of the coding manual, as well as to look for similarities or differences in the codes between the two data sets collected during each phase to allow for triangulation of these data. During this stage, it was found that the single coding manual could be effectively applied to both data sets, and, as such, the data from both were collated.

(d) Themes were then reviewed and refined by the research team. At this stage, differences in responses between subgroups were also examined by identifying instances where there was a notable difference in the number of voiced opinions between subgroups (age, gender, and cognitive score). Other subgroup comparisons based on educational level and frequency of Internet use were not carried out as participants were unequally distributed across several subgroups, resulting in small numbers of participants within most subgroups.

(e) The names attributed to both themes and subthemes were then refined to present a clear and concise reflection of the data, as well as to clearly relate them to the guiding principles.

(f) Finally, the results were written up in order to present a concise and coherent account of the findings in relation to the guiding principles.

\section{Results}

\section{Participant Characteristics}

In total, 52 participants were interviewed, with 34 taking part in think-aloud interviews, 11 taking part in retrospective interviews, 
Table 2 Participant Characteristics

\begin{tabular}{lc}
\hline Characteristic & \\
\hline Age (in years) & $70.3(5.3)$ \\
Mean $(S D)$ & $61-80$ \\
Range & \\
Gender & $30(58 \%)$ \\
Female & $22(42 \%)$ \\
Male & \\
Cognitive score & $26(50 \%)$ \\
Lower & $26(50 \%)$ \\
Higher & \\
Educational level & $5(10 \%)$ \\
No exams & $19(37 \%)$ \\
GCSC & $7(13 \%)$ \\
A level & $8(15 \%)$ \\
Undergraduate studies & $5(10 \%)$ \\
Postgraduate studies & $8(15 \%)$ \\
Other & \\
Frequency of Internet use & $39(75 \%)$ \\
Every day & $9(17 \%)$ \\
Few times per week & $1(2 \%)$ \\
Once every 2 weeks & $1(2 \%)$ \\
Once a month & $1(2 \%)$ \\
Hardly ever & $1(2 \%)$ \\
Unknown & \\
\hline
\end{tabular}

Note. Values are reported as $n(\%)$ unless specified.

and seven taking part in both (Table 2). The mean length of thinkaloud interviews was 95 min $(S D=17)$, with a median of 92 min and a range between 64 and $131 \mathrm{~min}$. The mean length of retrospective interviews was $30 \mathrm{~min}(S D=19)$, with a median of 26 min and a range between 10 and 91 min.

The findings are organized in four sections, discussing the extent to which participants' perceptions reflected each of the four guiding principles' key design objectives and associated intervention features.

\section{Design Objective: Encourage Engagement With and Intrinsic Motivation for Physical Activity}

Key intervention feature: Offer novel activities that are compatible with users' lifestyles and identities: Lifestyle activity, strength and balance training, breaks from sitting. Many users of both genders had positive reactions to the suggested activities, often commenting on how accessible they were, describing them as easy, useful, and practical. Men were, however, more likely than women to express positive views about the activities. Many expressed feeling encouraged to try them. 'I'm gonna do the walking bit, I'm gonna play golf, that'll be a good start, won't it?" (P0118, male, 70, lower cognitive score, think-aloud interview).

Some participants liked the idea of being encouraged to engage in everyday activities that they enjoy, instead of traditional exercise, explaining that because of this, it was more likely that users of the intervention would follow the suggestions. Some also spoke appreciatively about being able to fit the suggested activities into their daily routines. "If you can build something into your life through something you like and make an exercise of it, it's better for you because you don't notice that you're exercising, if you understand me?" (P0130, female, 75, lower cognitive score, thinkaloud interview).

Some people thought that the suggested activities were not relevant to them, mainly because they felt that they were already sufficiently physically active. Some, however, mentioned that using the intervention reminded them about the importance of being physically active, validating the activities they were already engaged in. "It's confirming what I have in my mind and I do try to do" (P0245, male, 66, lower cognitive score, think-aloud interview).

Key intervention feature: Rather than framing activities in terms of reducing risk of health conditions, highlight benefits that have immediately evident and noticeable outcomes and are known to be valued by the intended user group. Many participants valued being given information about the benefits of physical activity, often mentioning that they appreciated being presented with supporting research evidence. Some expressed increased motivation to become more physically active as a result. "Taking a short two minute walk every half hour improves people's blood sugar levels.' Oh, I've got terrible problems with blood sugars. Right. Good. Now I know what to do about it" (J0101, female, 62, higher cognitive score, think-aloud interview).

Without commenting on specific features of the intervention, a few participants discussed motivation and discipline being prerequisites to being physically active.

So I think that's the thing to be, have to be determined and to motivate yourself, 'cause ... I think it possibly is too easy, you know, being retired, oh it's nice and cosy inside so I'll sit inside and think about it, perhaps do it mañana as they say, tomorrow. (P0112, female, 68, lower cognitive score, thinkaloud interview)

\section{Design Objective: Minimize Risk of Users Receiving Activity Suggestions That Are Too Advanced and Unsafe or Too Basic and Which Do Not Increase Capacity; Steer Users Toward Those Activities That Seem Most Personally Suitable/Beneficial; Offer Users Choice and Autonomy Regarding Content and Activities They Engage With (Within Those Suitable).}

Key intervention feature: Advice tailored based on current activity levels and perceptions of current strength and balance skills. A few participants had positive reactions to being presented with different difficulty levels for strength and balance activities.

Yeah, I think you need to do that. ... Say if it didn't and it told me that I'd got to, you know, do this four times a day, I'd just get bored. And I wouldn't move on, or, you know, or I'd want to jump a load of it and get further on. So, I think tailoring it early on in the active bit is, yeah, it's essential. (J0104, male, 65 , higher cognitive score, think-aloud interview)

Key intervention feature: Offer a variety of activities to suit different levels of ability. Many people spoke positively about the fact that there are activities to suit everyone, allowing people with a wide range of abilities to start being more active. Some were pleased by the fact that the suggested activities seemed achievable 
by older adults. Participants with higher cognitive scores were more likely to voice this opinion than those with lower cognitive scores. Some speculated that proposing unreachable goals would likely put people off. "If you give people tasks that are just beyond, you know, just too unreasonable, you're switching them off and they won't bother doing it, totally" (P0111, male, 67, higher cognitive score, think-aloud interview).

\section{Design Objective: Build Confidence to Undertake More Activity}

Key intervention feature: Provide reassurance, encouragement, techniques, and strategies to reduce concerns and barriers: "Common Concerns" pages. Some participants had positive reactions to concerns about being physically active being discussed by the intervention. Some also valued seeing concerns about the safety aspects of engaging in physical activity being addressed, with some mentioning it would help with choosing and engaging in activities that are safe.

I think it gives you a bit of confidence to do it at your own pace ... in a safe environment without sort of doing these things in an environment where you might not be safe if things go wrong. So, I think it sort of puts the perspective in your mind of what you should be expecting to do. (P0130, female, 75, lower cognitive score, think-aloud interview)

Key intervention feature: Provide reassurance, encouragement, techniques, and strategies to reduce concerns and barriers: Provision of graded activities with gradual increases from low activity baseline. Some users described the importance of being able to choose their own activities and goals in order to start slowly and to progressively increase amounts of physical activity over time.

You are making your own plan. The thing is to get you into the game and then, as the game goes on, then, "Oh, I could do ten easy," then I'll up it or, yes, no, that's good because you are in control. (P0211, male, 73, lower cognitive score, think-aloud interview)

Key intervention feature: Provide reassurance, encouragement, techniques, and strategies to reduce concerns and barriers: Stories from similar others about their successes with building up activities. A majority of participants expressed liking the "success stories," specifically commenting on the fact that they were relatable and helped with seeing how others have successfully increased their physical activity levels from a similar starting point. Participants in their sixties were more likely to comment on this than those in their seventies and eighties.

In both cases, you've chosen someone who's probably less active with Jo, and then someone who in the past was a little bit more active with Elsie, but is now finding that-which is a bit like me with my knees, I just find it's hard to run, really. (J0104, male, 65, higher cognitive score, think-aloud interview)

A few people had more ambivalent feelings about the "success stories," explaining that they are not interested in them, or that they would not help them with choosing activities.

I'm not really keen on the stories, to be honest. I mean, it might help people ... I don't know. I think if you want to do something you will do it anyway. You don't really need other people to say how you can manage it. If you wanna do something, you will do it. (J0112, female, 61, higher cognitive score, think-aloud interview)

Key intervention feature: Provide reassurance, encouragement, techniques, and strategies to reduce concerns and barriers: Access to a trained supporter to support engagement. Participants in this study did not have access to a trained supporter (a feature of the planned larger trial), but a few liked the idea of having access to one (by either telephone or e-mail) to help them start engaging with the suggested activities.

Oh, well, it's lovely when someone takes an interest in how you're getting on. And it helps to motivate you. A, because you want to have good news to tell them, and B, because the process of having to give the feed-back makes you more aware of what you're slipping on and what you're not slipping on.... If it was a bit more specific, you know, sort of "okay, what have you found have been the obstacles?" So something to help you think through what would help you keep going on it, and what would improve it. Would be really useful, a bit of encouragement. (J0101, female, 62, higher cognitive score, think-aloud interview)

A small number of users did not feel the need for a support person, explaining that the intervention appeared sufficiently clear for them to engage with the intervention's proposed activities independently, or that support is not something they would seek. "I'm quite a loner actually. I don't really look for support. I stick my heels in and get on with it. I don't look for that support from anyone in particular" (P0106, male, 76, lower cognitive score, think-aloud interview).

Key intervention feature: Provide reassurance, encouragement, techniques and strategies to increase self-efficacy: Graded goal setting and review. Many people spoke positively about the intervention's goal setting features and some discussed benefits, such as helping with habit forming or avoiding participants drifting from their plans. A small number of participants commented on the fact that setting goals is a form of commitment, explaining that this would help them with keeping to their goals.

Yes, I think it's a good idea because it gives you an objective, doesn't it, to aim at. If you think, I'll have a walk this morning. You think, it's raining, I'll wait until tomorrow, but if you've got it there in black and white. You said you're going to do it then you should really try and do it. (P0106, male, 76, lower cognitive score, think-aloud interview)

Some participants had more negative feelings about setting goals, citing a variety of reasons: not liking lists; feeling like activities would become a chore; not liking the added pressure setting goals would apply; preferring to take each day as it comes; or not feeling sufficiently disciplined to achieve their goals.

Setting the goals is good, but you, kind of, forget about goals, anyhow, don't you? You say, I'll do that every time but, no, it never really quite works. I don't have the discipline to do it, every time I should do. (P0261, male, 62, higher cognitive score, retrospective interview)

Many users mentioned being pleased with being able to look back and review activity goals and progress. Some added that seeing improvements would give a sense of achievement and motivate them to continue. 
Well, that does sound good that you can sort of see where you are and where you've got to and sort of think "I couldn't do that so many weeks ago." So it's nice that you can actually got something that you can go back and assess it by. (J0112, female, 61, higher cognitive score, think-aloud interview)

\section{Design Objective: Minimize Cognitive Load and Dependence on the Intervention Website}

Key intervention feature: Suggestions and examples of how to create new physical activity habits. A few participants discussed the importance of habit forming and were pleased with how the suggested ideas about incorporating physical activity into daily routines might help with this. For some, this also inspired them to think about other ways in which they could incorporate more physical activity.

Then you'll think, "Oh yes, well ... instead of taking all the clean washing upstairs in one go, I'll take it in two gos." And when I go up the stairs, I'll go a bit slower, because that's better exercise, go up it a bit slower. Or when I'm ... well, you know, when I'm doing whatever, I'll do a few more extra, you know, a few extras of this, or I'll go a bit further. (P0112, female, 68, lower cognitive score, think-aloud interview)

Key intervention feature: Suggestions and examples of how to make small changes to everyday physical and social environments to prompt activity. A few people had positive reactions to the environmental restructuring suggestions, particularly those that involved placing everyday items away from usual locations.

I think putting the TV remote on the mantelpiece or next to the TV is a really good one 'cause I get so conscious some evenings that I'm sitting there on the sofa with the remote next to me and, actually, just to leave it by the telly, if I wanted to change channel, I think that's a really good one. (J0105, female, 65, higher cognitive score, think-aloud interview)

A few participants expressed not liking or wanting to follow the environmental restructuring suggestions, explaining that they would find them impractical. "Because when I want to sit still I want to sit still, and I don't want to get up to get the remote off the mantelpiece or to get the drink that I left further away, you know?" (P0105, female, 76, higher cognitive score, think-aloud interview).

Many users spoke about how involving others would make them more inclined to engage in physical activity, by increasing enjoyment, encouragement, and motivation. A few also mentioned that organizing activities with others would likely help them keep to their plans.

If you agree to meet up with someone and do something, then it does make you think, well yes, I've got to do that because I said I'll meet them at such and such a time and I'll do something or they're coming around and we're going to do something. Whereas if you don't do that, it's easy to think, oh, I'll do it later and then not do it. (P0269, female, 64, lower cognitive score, retrospective interview)

Social support seemed to be a matter of personal preference as a few participants felt that they would be happy doing activities on their own and did not feel the need to involve others. "I'm quite happy with my own company" (P0129, female, 67, lower cognitive score, retrospective interview).
Key intervention feature: Support for planning environmental prompting for each activity goal set (time of day and links to specific activities). A few participants valued being able to specify a detailed plan for their goals, with some explaining that it would also increase the likelihood of doing their chosen activities.

Yes, well I think you must have a plan otherwise you're not going to stick to a routine, are you, and you're never going to do it properly. If you say you're going to do it for ten minutes or quarter of an hour at a certain time, and you do it every day like that, it's a good idea. Otherwise, it'll just fall by the wayside, I think, won't it? (P0106, male, 76, lower cognitive score, think-aloud interview)

Key intervention feature: Exercise instructions/goals set/record sheets available as printouts. Many people discussed the benefits of being able to print their goals, including using printouts as convenient visual reminders, avoiding the need to use their computer to access their goals.

And also, I mean, it's, you know, if it's there on a bit of paper, you can pin it up on the wall in the kitchen, and, "Oh yeah, I need to do that today," you know, rather than get the laptop out and go online or what have you. (J0114, male, 70, higher cognitive score, think-aloud interview)

Key intervention feature: Signposting/links to offline resources to support activity. A small number of participants commented on this feature, with positive reactions about being able to access further advice and information about additional activities and how to access them locally.

\begin{abstract}
“'Useful links.' Oh, that's good, 'Information on walking groups.' ... And Tai Chi. So again, it's good that you've got link - and I presume this will be somewhere to phone or a website." (P0142, female, 62, higher cognitive score, thinkaloud interview)
\end{abstract}

\section{Discussion}

The main aim of this study was to explore older adults' perceptions of Active Lives with regard to how acceptable they found its intervention features. In doing so, we also examined the extent to which participants' experiences of using Active Lives resonated with the theory- and evidence-based guiding principles that informed the intervention content and format. Our findings are encouraging in that participants mainly expressed positive perceptions of the intervention's key features. For the most part, participant views were in accordance with the expected intervention mechanisms outlined in the guiding principles, the purpose of which aimed to maximize engagement and acceptability. Our findings also identified some features that were not appreciated by all participants, and which should therefore be cautiously implemented as optional content, as well as identifying participants' perceived need for additional content.

Participants valued the features derived from the intervention's first guiding principle, which aimed to encourage intrinsic motivation for, and engagement with, physical activity. The participants appreciated the variety and accessibility of the suggested activities, and that these could be integrated into daily routines without difficulty. Our findings concur with existing literature about the type of activities that should be encouraged with this age group, as many older adults prefer to remain physically active through 
everyday activities, as opposed to engaging in physical activity as a purposeful activity within itself (McGowan, Devereux-Fitzgerald, Powell, \& French, 2018). The fact that the activities suggested by Active Lives can be carried out close to home and do not involve significant costs may have further contributed to their appeal, as these are factors that have been found to positively affect the acceptability of physical activities by many older adults (DevereuxFitzgerald et al., 2016).

Information about the immediate benefits of physical activity was also well received, with participants appreciating that this was accompanied by supporting research evidence. Our participants' views align with previous literature about older adults appreciating present-oriented benefits (those in Active Lives included keeping mobile, maintaining independence, enjoyment, reducing pain, social connection, enhancing mood, and maintaining general quality of life), as opposed to long-term health benefits (McGowan et al., 2018).

This study also allowed us to identify additional features that would be beneficial for encouraging intrinsic motivation for physical activity. A few participants discussed motivation as being something important to consider in itself, which suggests that including topics on ways to remain motivated would likely benefit users who may struggle with this. As many of the motivational techniques we had implemented were implicit in nature (e.g., providing choice and using nondirective language, or presenting enjoyable activities and associated short-term benefits), this finding highlighted the need for more explicit motivational strategies to be included. As a result, supplementary content that discusses ways to remain motivated was subsequently implemented. This included additional pages and downloadable sheets addressing topics such as habit forming (Lally \& Gardner, 2013), enjoyment and involving others (Devereux-Fitzgerald et al., 2016), remembering successes as a means to increase self-efficacy (Bandura 1977; Schutzer \& Graves, 2004), and exploring personal reasons for being physically active, in order to help users identify intrinsic motivating factors (Deci \& Ryan, 1985; Ryan \& Deci, 2000). Further relating to our guiding principle to build intrinsic motivation for physical activity, some participants reported feeling that they were already sufficiently physically active and explained that this limited the extent to which they thought they would find the intervention's suggested activities engaging or motivating. To try and address this, suggestions were added for users to include their current activities when setting goals in order to build on activities they already did and enjoyed, as well as a message suggesting that no matter the activity level, it would be good to try and do a little bit more.

Features associated with the intervention's second guiding principle - to ensure there was a wide range of activity suggestions to suit older adults of all levels of ability-were also valued by many of our participants. As older adults report doubts about their abilities to engage in physical activity (McGowan et al., 2018), and have concerns about falling and sustaining injuries during physical activity (Franco et al., 2015), our findings suggest that offering activities that cover a range of abilities is an acceptable way of minimizing these barriers.

Our participants appreciated features resulting from the intervention's third guiding principle - to build users' confidence to engage with physical activity. Features to address this objective included "success stories," which were positively commented on by a majority of participants. These "success stories" challenge commonly held perceptions by older adults that engaging in physical activity is not compatible with their self-identity (McGowan et al.,
2018). Our participants' positive comments about these "success stories" may reflect recognition that this feature could help older adults reconsider their ability and confidence to try some of the recommended activities and, in doing so, could inspire a renewed sense of enthusiasm for physical activity. The individuals portrayed in the Active Lives "success stories" are all older adults who have made small, manageable increases in physical activity, for instance, doing a little more walking and using stairs instead of lifts, helping a less able friend with gardening, or standing up and stretching during TV ads. As such, our participants' views on these stories are consistent with research findings in the area of role modeling and physical activity in later years, which provide indications that for moderately or inactive older adults, the most effective role models may be peers who are slightly more physically active than them (Horton, Dionigi, \& Bellamy, 2013), as opposed to highly physically active older adults or those with elite athlete Masters status, whose achievements may be perceived as intimidating and unrealistic (Horton et al., 2013; Horton, Baker, Cote, \& Deakin, 2008; Ory, Kinney Hoffman, Hawkins, Sanner, \& Mockenhaupt, 2003). A few participants were, however, more ambivalent about this feature, explaining that they did not feel the need to read other's stories to engage with the intervention or the suggested activities. This finding provided evidence for the need for "success stories" to be included as optional content, as originally designed.

A further feature that aimed to build users' confidence was goal setting and reviewing. Goal setting and reviewing were among the most discussed features, with some participants commenting on how these would likely increase their motivation to engage in physical activity. Previous studies have reported mixed findings with regard to the use of self-regulatory techniques, such as goal setting and reviewing with different age groups (French et al., 2014; Michie, Abraham, Whittington, McAteer, \& Gupta, 2009; Williams \& French, 2011). French et al. (2014) suggested that older adults may experience self-regulatory features, such as goal setting and reviewing, as burdensome tasks due to declining executive function (involved in forming and implementing intentions). This does not resonate with our participants' experiences of these features, who infrequently expressed negative views about these features. These conflicting findings could be due, in part, to these features being implemented in a variety of ways across different interventions, with varying levels of success, that is, there may be implementation failure in some studies (Cargo et al., 2018). It may be that these features were more acceptable within Active Lives because they were designed to be accessible and easy to use, with many goal suggestions and planning features to select from in an attempt to reduce cognitive demands as much as possible. When participants did not like goal-related features, there was no indication that this was due to difficulties with the required cognitive effort to use them, as our participants' negative perceptions of these features included their dislike of lists, the unwanted added pressure of reaching goals, and not feeling sufficiently disciplined. Furthermore, the subgroup analysis did not show any substantial differences in views between people with lower and higher cognitive scores. This suggests that engagement with self-regulatory techniques may not be dependent on cognitive ability. Some of those who disliked these features provided rationales that suggest that these individuals may be averse to structure or to making plans, which may reflect an attachment to a more unconstrained lifestyle enjoyed in later years, when the need to plan around parental responsibilities or full-time work is often no longer required (French et al., 2014). As negative perceptions of goal-related features were infrequent, these features were retained in their 
original form; users are encouraged to try them the first time they encounter them, but they are presented as optional content in later sessions, since they are not suitable for all users, as illustrated by our findings.

Features related to the intervention's fourth guiding principleto minimize cognitive load and dependence on the intervention website-were also valued by our participants. They were particularly pleased with being able to use printouts of their goals as visual prompts and, in doing so, avoiding having to use a device to access the intervention to remind themselves of their chosen activities and goals, further reducing the cognitive effort involved in following up on their goals. This extends the literature as we could not find any reports on user perspectives on goal printing features in the context of similar physical activity interventions for older adults. As such, this finding may be of use for the development of future digital physical activity interventions.

Another successful feature relating to the fourth guiding principle was intervention content encouraging the involvement of others. This was discussed by many participants, who mentioned important benefits such as increased enjoyment, encouragement, and motivation to engage in physical activity. These views agree with previous findings about older adults generally appreciating being physically active with others and valuing the motivation to persist this provides through enjoyment of social interactions (Devereux-Fitzgerald et al., 2016; Franco et al., 2015; McGowan et al., 2018). A few participants mentioned preferring to independently engage with the activities, which highlights the need for this feature to be optional and presented as a suggestion, which may help prevent potential disengagement from the intervention and its suggested activities by the minority of users who may not value being physically active with others.

Overall, our participants' perceptions of the intervention components of Active Lives are encouraging and have allowed us to optimize the intervention content during its development. From a wider perspective, our participants' positive views suggest that the overall approach to physical activity used by Active Lives has potential for being more successful than other approaches that focus on prescriptive guidelines, disease prevention narratives, and framing physical activity solely as a means to transform an aging body into a fit, more performant one. These strategies are associated with health policies resulting from the concept of active aging (World Health Organization, 2002). Active aging was designed to counter the more prevalent narrative of decline (which portrays the inevitable deterioration of aging bodies; Gullette, 1997). Active aging has subsequently been criticized as contributing to a "war on old age" (Vincent, 2007), with those not living up to the standards of active (and successful) aging implicitly failing, with negative consequences on their perceptions of self (Holstein \& Minkler, 2007). While Active Lives still aims to counter the narrative of decline, it does so by using a nondirective approach devoid of guidelines based on minimum amounts of time for physical activity and by instead suggesting activities accessible to most (including those with low incomes or limited time) that can be integrated into everyday routines. Active Lives provides a variety of ideas for increasing physical activity and, in doing so, implicitly acknowledges that older adults' lives are diverse, encouraging users to make sense of how physical activity might fit within their current lifestyle and abilities. Active Lives focuses on the immediate benefits of physical activity (keeping mobile, maintaining independence, enjoyment, reducing pain, social connection, enhancing mood, and maintaining general quality of life), with a view to enriching the lives of older adults, a more positive and engaging approach to implementing active aging interventions, as put forward by Phoenix and Tulle (2017).

The intervention is being trialled in a feasibility study (in 2019-2020), and a large trial $(n=21,455)$ is being planned to evaluate Active Lives' intended behavioral outcomes over a period of 5 years as part of the Active Brains web-based intervention. This trial will help to determine whether Active Lives is effective in increasing and maintaining older adults' levels of physical activity over the long term.

\section{Limitations}

Using qualitative interviews enabled a detailed exploration of individual perceptions and experiences of the features of Active Lives through interactive and adaptable questioning. However, some participants may have been reluctant to express negative views to the interviewers (despite being encouraged to do so before and during interviews), due to acquiescence and social desirability biases (Bowling, 2014). It is also possible that the nonresponse bias (Bowling, 2014) may have played a role in shaping the sample, with those who chose to participate being more motivated than those who did not; although, this is not always the case with this type of research, for example, Corbett, Cheetham, et al. (2018).

Our sample was large and diverse in terms of lower/higher cognitive scores and educational level. However, we did not capture participants' ethnic backgrounds. Recruitment of participants was limited to the south coast of England, and most participants appeared to be White British. It is therefore unclear whether findings are transferable to those from other ethnic groups. For this reason, as part of the planned nationwide trial, recruitment from GP practices covering areas that include a significant proportion of people who do not identify as White will be purposefully increased. Recruitment from practices in areas with lower indices of multiple deprivation will also be increased in order to ensure a diverse sample in terms of socioeconomic status.

All participants in our sample already had access to the Internet, and a majority used it daily. It is therefore possible that views from inexperienced computer/Internet users may vary from those expressed by our participants.

\section{Implications}

The present research has important implications for other developers of physical activity digital interventions for older adults. Our findings provide insights into this group's particular needs in terms of their motivations, values, abilities, and concerns regarding physical activity. Among the features our participants appreciated the most, findings related to three of them merit greater attention, as they extend the literature and seem particularly relevant to this age group. The inclusion of goal setting and reviewing features has been the object of mixed findings as there have been suggestions that their use may be disliked or too burdensome for older adults. Our findings suggest such features may be useful and valued by many people in this age group, provided they are presented as optional content (as not appreciated by all) and implemented in such a way that minimizes cognitive demands. Second, the portrayal of older individuals who have made small, manageable increments to their physical activity levels within the optional "success stories" feature has been well received by a majority of participants. This provides further evidence that role modeling content consisting of peers who are slightly more active may be an 
effective strategy for engaging older adults who are not highly physically active. Third, the ability for users to print their goals for offline access, a novel feature not previously discussed in the literature, was also appreciated by many participants. This suggests that including this feature in the design of physical activity interventions destined for older adults may be useful; as in the context of Active Lives, participants spoke about how this would help them remember and act on their chosen goals.

\section{Conclusion}

Using the person-based approach to develop a digital intervention to promote physical activity in older adults enabled us to identify and adopt design features that our target user group found broadly appealing. Our participants' views were generally in agreement with the guiding principles that informed the intervention design, as well as with previous literature regarding factors that influenced the uptake of physical activity by older adults. Where they were not, this helped us to identify areas where additional features and techniques may enhance the acceptability of intervention content. These insights into the features and elements that older adults appreciated builds on the resources available to other digital intervention developers. While our findings are promising, a fully powered 5-year trial is being planned to investigate how effective Active Lives might be with increasing and maintaining engagement with physical activity in the longer term.

\section{Acknowledgments}

This work was supported by the National Institute for Health Research under its Programme Grants for Applied Research (reference RP-PG0615-20014). The views expressed are those of the authors and not necessarily those of the National Institute for Health Research or the Department of Health and Social Care. The Active Lives intervention was developed using LifeGuide software, which was partly funded by the National Institute for Health Research Southampton Biomedical Research Centre. The authors would like to thank Patient and Public Involvement representatives Rosemary Phillips, Bernard Gudgin, John Niven, and Tom Stokes, who provided feedback on the design of the intervention.

\section{References}

Aarts, H., Paulussen, T., \& Schaalma, H. (1997). Physical exercise habit: On the conceptualization and formation of habitual health behaviours. Health Education Research, 12(3), 363-374. PubMed ID: 10174219 doi:10.1093/her/12.3.363

Ammann, R., Vandelanotte, C., de Vries, H., \& Mummery, W.K. (2013). Can a website-delivered computer-tailored physical activity intervention be acceptable, usable, and effective for older people? Health Education \& Behavior, 40(2), 160-170. PubMed ID: 23077157 doi: 10.1177/1090198112461791

Antonucci, T.C., Ashton-Miller, J.A., Brant, J., Falk, E.B., Halter, J.B., Hamdemir, L., ... Webster, N.J. (2012). The right to move: A multidisciplinary lifespan conceptual framework. Current Gerontology and Geriatrics Research, 2012, 873937. PubMed ID: 23251148 doi:10.1155/2012/873937

Baddeley, A.D. (1968). A 3 min reasoning test based on grammatical transformation. Psychonomic Science, 10(10), 341-342. doi:10.3758/ BF03331551
Bandura, A. (1977). Self-efficacy-Toward a unifying theory of behavioral change. Psychological Review, 84(2), 191-215. PubMed ID: 847061 doi:10.1037/0033-295X.84.2.191

Bandura, A. (1986). Social foundations of thought and action: A social cognitive theory. Upper Saddle River, NJ: Prentice-Hall.

Bandura, A. (1991). Social cognitive theory of self-regulation. Organizational Behavior and Human Decision Processes, 50(2), 248-287. doi:10.1016/0749-5978(91)90022-L

Bandura, A. (2012). On the functional properties of perceived self-efficacy revisited. Journal of Management, 38(1), 9-44. doi:10.1177/ 0149206311410606

Bennett, G.G., \& Glasgow, R.E. (2009). The delivery of public health interventions via the internet: Actualizing their potential. Annual Review of Public Health, 30(1), 273-292. PubMed ID: 19296777 doi:10.1146/annurev.publhealth.031308.100235

Bowling, A. (2014). Research methods in health: Investigating health and health services (4th ed.). Maidenhead, UK: Open University Press.

Braun, V., \& Clarke, V. (2006). Using thematic analysis in psychology. Qualitative Research in Psychology, 3(2), 77-101. doi:10.1191/ 1478088706qp063oa

Bravata, D.M., Smith-Spangler, C., Sundaram, V., Gienger, A.L., Lin, N., Lewis, R., ... Sirard, J.R. (2007). Using pedometers to increase physical activity and improve health-A systematic review. JAMA, 298(19), 2296-2304. doi:10.1001/jama.298.19.2296

Buchman, A.S., Boyle, P.A., Yu, L., Shah, R.C., Wilson, R.S., \& Bennett, D.A. (2012). Total daily physical activity and the risk of AD and cognitive decline in older adults. Neurology, 78(17), 1323-1329. PubMed ID: 22517108 doi:10.1212/WNL.0b013e3182535d35

Cargo, M., Harris, J., Pantoja, T., Booth, A., Harden, A., Hannes, K., . . . Noyes, J. (2018). Cochrane Qualitative and Implementation Methods Group guidance series-paper 4: Methods for assessing evidence on intervention implementation. Journal of Clinical Epidemiology, 97, 59-69. PubMed ID: 29223325 doi:10.1016/j.jclinepi.2017. 11.028

Charters, E. (2003). The use of think-aloud methods in qualitative research an introduction to think-aloud methods. Brock Education, 12(2), $68-82$.

Clarke, T.C., Norris, T., \& Schiller, J.S. (2017). Early release of selected estimates based on data from 2016 National Health Interview Survey. National Center for Health Statistics. Retrieved from https://www. cdc.gov/nchs/data/nhis/earlyrelease/Earlyrelease201705.pdf

Corbett, T., Cheetham, T., Muller, A.M., Slodkowska-Barabasz, J., Wilde, L., Krusche, A., .. Bradbury, K. (2018). Exploring cancer survivors' views of health behaviour change: "Where do you start, where do you stop with everything?" Psycho-Oncology, 27(7), 1816-1824. doi:10.1002/pon.4732

Corbett, T., Singh, K., Payne, L., Bradbury, K., Foster, C., Watson, E., .. . Yardley, L. (2018). Understanding acceptability of and engagement with Web-based interventions aiming to improve quality of life in cancer survivors: A synthesis of current research. Psycho-Oncology, 27(1), 22-33. doi:10.1002/pon.4566.

Corder, K., Ogilvie, D., \& van Sluijs, E.M. (2009). Invited commentary: Physical activity over the life course-Whose behavior changes, when, and why? American Journal of Epidemiology, 170(9), 10781081. PubMed ID: 19767350 doi:10.1093/aje/kwp273

Crabtree, B.F., \& Miller, W.L. (Eds.). (1999). Doing qualitative research (2nd ed). Thousand Oaks, CA: SAGE publications.

Craig, C.L., Marshall, A.L., Sjostrom, M., Bauman, A.E., Booth, M.L., Ainsworth, B.E., ... Oja, P. (2003). International physical activity questionnaire: 12 -country reliability and validity. Medicine \& Science in Sports \& Exercise, 35(8), 1381-1395. PubMed ID: 12900694 doi: 10.1249/01.MSS.0000078924.61453.FB 
Davies, C.A., Spence, J.C., Vandelanotte, C., Caperchione, C.M., \& Mummery, W.K. (2012). Meta-analysis of internet-delivered interventions to increase physical activity levels. International Journal of Behavioral Nutrition and Physical Activity, 9(1), 52. PubMed ID: 22546283 doi:10.1186/1479-5868-9-52

Deci, E., \& Ryan, R.M. (1985). Intrinsic motivation and self-determination in human behavior. New York, NY: Plenum.

Dennison, L., Morrison, L., Lloyd, S., Phillips, D., Stuart, B., Williams, S., ... Yardley, L. (2014). Does brief telephone support improve engagement with a web-based weight management intervention? Randomized controlled trial. Journal of Medical Internet Research, 16(3), e95. doi:10.2196/jmir.3199

Department of Health and Social Care. (2019). UK chief medical officers' physical activity guidelines. Retrieved from https://assets.publishing. service.gov.uk/government/uploads/system/uploads/attachment_data/ file/832868/uk-chief-medical-officers-physical-activity-guidelines.pdf

Devereux-Fitzgerald, A., Powell, R., Dewhurst, A., \& French, D.P. (2016). The acceptability of physical activity interventions to older adults: A systematic review and meta-synthesis. Social Science \& Medicine, 158, 14-23. PubMed ID: 27104307 doi:10.1016/j.socscimed.2016. 04.006

Essery R., Pollet S., Smith K.A., Mowbray, F., Slodkowska-Barabasz, J., Denison-Day, J., ... Yardley, L. (2020). Planning and optimising a digital intervention to reduce older adults' cognitive decline. Research Square. Advance online publication. doi:10.21203/rs.3. rs-20513/v1

Franco, M.R., Tong, A., Howard, K., Sherrington, C., Ferreira, P.H., Pinto, R.Z., \& Ferreira, M.L. (2015). Older people's perspectives on participation in physical activity: A systematic review and thematic synthesis of qualitative literature. British Journal of Sports Medicine, 49(19), 1268-1276. doi:10.1136/bjsports-2014-094015

French, D.P., Olander, E.K., Chisholm, A., \& Mc Sharry, J. (2014). Which behaviour change techniques are most effective at increasing older adults' self-efficacy and physical activity behaviour? A systematic review. Annals of Behavioral Medicine, 48(2), 225-234. PubMed ID: 24648017 doi:10.1007/s12160-014-9593-z

Fylan, F. (2005). Semi-structured interviewing. In J. Miles \& P. Gilbert (Eds.), A handbook of research methods for clinical and health psychology (p. xi, 315 p.). New York, NY: Oxford University Press.

Gilgun, J.F. (2013). Grounded theory, deductive qualitative analysis, and social work research and practice. In A.E. Fortune, W. J. Reid, \& R. L. Miller, Jr. (Eds.), Qualitative research in social work (Vol. 107, pp. 107-135). New York, NY: Columbia University Press.

Gullette, M.M. (1997). Declining to decline: Cultural combat and the politics of the midlife. Charlottesville; London: University Press of Virginia.

Health and Social Care Information Centre. (2017). Health Survey for England 2016: Physical activity in adults. Retrieved from http:// healthsurvey.hscic.gov.uk/media/63730/HSE16-Adult-phy-act.pdf

Holstein, M.B., \& Minkler, M. (2007). Critical gerontology: reflections for the 21 st century. In M. Bernard \& T. Scharf (Eds.), Critical perspectives on aging societies (pp. 13-26). Cambridge, UK, Polity Press.

Horton, S., Baker, J., Cote, J., \& Deakin, J.M. (2008). Understanding seniors' perceptions and stereotypes of aging. Educational Gerontology, 34(11), 997-1017. doi:10.1080/03601270802042198

Horton, S., Dionigi, R., \& Bellamy, J. (2013). Women aged 75 and over: Attitudes towards health-related role models and female master athletes. International Journal of Interdisciplinary Social and Community Studies, 7(3). 33-47. doi:10.18848/2324-7576/CGP/ v07i03/53448.

Kohl, H.W., Craig, C.L., Lambert, E.V., Inoue, S., Alkandari, J.R., Leetongin, G., ... Workin, L.P.A.S. (2012). The pandemic of physical inactivity: Global action for public health. The Lancet, 380(9838), 294-305. PubMed ID: 22818941 doi:10.1016/S01406736(12)60898-8

Kohl, L.F., Crutzen, R., \& de Vries, N.K. (2013). Online prevention aimed at lifestyle behaviors: A systematic review of reviews. Journal of Medical Internet Research, 15(7), e146. doi:10.2196/jmir.2665

Koorts, H., Eakin, E., Estabrooks, P., Timperio, A., Salmon, J., \& Bauman, A. (2018). Implementation and scale up of population physical activity interventions for clinical and community settings: The PRACTIS guide. International Journal of Behavioral Nutrition and Physical Activity, 15(1), 51. PubMed ID: 29884236 doi:10.1186/ s12966-018-0678-0

Lachman, M.E., Lipsitz, L., Lubben, J., Castaneda-Sceppa, C., \& Jette, A.M. (2018). When adults don't exercise: Behavioral strategies to increase physical activity in sedentary middle-aged and older adults. Innovation in Aging, 2(1), 1-12. doi:10.1093/geroni/igy007

Lally, P., \& Gardner, B. (2013). Promoting habit formation. Health Psychology Review, 7, S137-S158. doi:10.1080/17437199.2011.603640

Lorig, K.R., \& Holman, H.R. (2003). Self-management education: History, definition, outcomes, and mechanisms. Annals of Behavioral Medicine, 26(1), 1-7. PubMed ID: 12867348 doi:10.1207/ S15324796ABM2601_01

Madkins, K., Moskowitz, D.A., Moran, K., Dellucci, T.V., \& Mustanski, B. (2019). Measuring acceptability and engagement of the keep it up! Internet-based HIV prevention randomized controlled trial for young men who have sex with men. AIDS Education and Prevention, 31(4), 287-305. PubMed ID: 31361516 doi:10.1521/aeap. 2019.31.4.287.

McGowan, L.J., Devereux-Fitzgerald, A., Powell, R., \& French, D.P. (2018). How acceptable do older adults find the concept of being physically active? A systematic review and meta-synthesis. International Review of Sport and Exercise Psychology, 11(1), 1-24. doi: 10.1080/1750984X.2016.1272705

Michie, S., Abraham, C., Whittington, C., McAteer, J., \& Gupta, S. (2009). Effective Techniques in Healthy Eating and Physical Activity Interventions: A meta-regression. Health Psychology, 28(6), 690-701. PubMed ID: 19916637 doi:10.1037/a0016136

Michie, S., Atkins, L., \& West, R. (2014). The Behaviour Change Wheel: A Guide to Designing Interventions. Sutton, UK: Silverback Publishing.

Michie, S., Richardson, M., Johnston, M., Abraham, C., Francis, J., Hardeman, W., ... Wood, C.E. (2013). The behavior change technique taxonomy (v1) of 93 hierarchically clustered techniques: Building an international consensus for the reporting of behavior change interventions. Annals of Behavioral Medicine, 46(1), 81-95. PubMed ID: 23512568 doi:10.1007/s12160-013-9486-6

Moore, G.F., Audrey, S., Barker, M., Bond, L., Bonell, C., Hardeman, W., ... Baird, J. (2015). Process evaluation of complex interventions: Medical Research Council guidance. BMJ, 2015, 350. doi:10. 1136/bmj.h1258

Morrison, L.G., Muller, I., Yardley, L., \& Bradbury, K. (2018). The person-based approach to planning, optimising, evaluating and implementing behavioural health interventions. The European Health Psychologist, 20(3), 464-469.

Morrison, L.G., Yardley, L., Powell, J., \& Michie, S. (2012). What design features are used in effective e-health interventions? A review using techniques from critical interpretive synthesis. Telemedicine and E-Health, 18(2), 137-144. PubMed ID: 22381060 doi:10.1089/ tmj.2011.0062

Muellmann, S., Forberger, S., Mollers, T., Broring, E., Zeeb, H., \& Pischke, C.R. (2016). EHealth interventions for the promotion of physical activity in older adults: A systematic review. European Journal of Public Health, 26, 50-50. doi:10.1093/eurpub/ckw165.023. 
National Institute on Aging. (2019). Go4Life. Retrieved from https:// go4life.nia.nih.gov/

Norton, S., Matthews, F.E., Barnes, D.E., Yaffe, K., \& Brayne, C. (2014). Potential for primary prevention of Alzheimer's disease: An analysis of population-based data (vol 13, pg 788, 2014). Lancet Neurology, 13(11), 1070-1070.

Notthoff, N., Klomp, P., Doerwald, F., \& Scheibe, S. (2016). Positive messages enhance older adults' motivation and recognition memory for physical activity programmes. European Journal of Ageing, 13(3), 251-257. PubMed ID: 28804382 doi:10.1007/s10433-0160368-1

O'Cathain, A., Croot, L., Duncan, E., Rousseau, N., Sworn, K., Turner, K.M., ... Hoddinott, P. (2019). Guidance on how to develop complex interventions to improve health and healthcare. BMJ Open, 9(8), e029954. PubMed ID: 31420394

Office for National Statistics. (2019). Internet users, UK: 2019. Retrieved from https://www.ons.gov.uk/businessindustryandtrade/itandinternet industry/bulletins/internetusers/2019

Oinas-Kukkonen, H., \& Harjumaa, M. (2008, February). Towards deeper understanding of persuasion in software and information systems. Presented at the First international IEEE Conference on Advances in Computer-Human Interaction (pp. 200-205).

Oinas-Kukkonen, H., \& Harjumaa, M. (2009). Persuasive systems design: Key issues, process model, and system features. Communications of the Association for Information Systems, 24(1), 28.

Ory, M., Kinney Hoffman, M., Hawkins, M., Sanner, B., \& Mockenhaupt, R. (2003). Challenging aging stereotypes: Strategies for creating a more active society. American Journal of Preventive Medicine, 25(3), 164-171. doi:10.1016/S0749-3797(03)00181-8

Petersen, R.C. (2016). Mild cognitive impairment. Continuum, 22(2 Dementia), 404-418.

Phoenix, C., \& Tulle, E. (2017). Physical activity and ageing. In J. Piggin, L. Mansfield, \& M. Weed (Eds.), The Routledge handbook of physical activity policy and practice (Chapter 18; pp. 264-274). London, UK: Routledge.

Powell, K.E., Paluch, A.E., \& Blair, S.N. (2011). Physical activity for health: What kind? How much? How intense? On top of what? Annual Review of Public Health, 32(1), 349-365. PubMed ID: 21128761 doi:10.1146/annurev-publhealth-031210-101151.
Ryan, R.M., \& Deci, E.L. (2000). Self-determination theory and the facilitation of intrinsic motivation, social development, and wellbeing. The American Psychologist, 55(1), 68-78. PubMed ID: 11392867 doi:10.1037/0003-066X.55.1.68.

Schutzer, K.A., \& Graves, B.S. (2004). Barriers and motivations to exercise in older adults. Preventive Medicine, 39(5), 1056-1061. PubMed ID: 15475041 doi:10.1016/j.ypmed.2004.04.003.

Sekhon, M., Cartwright, M., \& Francis, J.J. (2017). Acceptability of healthcare interventions: An overview of reviews and development of a theoretical framework. BMC Health Services Research, 17(1), 88. PubMed ID: 28126032 doi:10.1186/s12913-017-2031-8.

Stockwell, S., Schofield, P., Fisher, A., Firth, J., Jackson, S.E., Stubbs, B., \& Smith, L. (2019). Digital behavior change interventions to promote physical activity and/or reduce sedentary behavior in older adults: A systematic review and meta-analysis. Experimental Gerontology, 120, 68-87. PubMed ID: 30836130 doi:10.1016/j.exger.2019.02.020.

Vincent, J. (2007). Science and imagery in the 'war on old age'. Ageing and Society, 27(6), 941-961. doi:10.1017/S0144686X07006630

Williams, S.L., \& French, D.P. (2011). What are the most effective intervention techniques for changing physical activity self-efficacy and physical activity behaviour-and are they the same? Health Education Research, 26(2), 308-322. PubMed ID: 21321008 doi: 10.1093/her/cyr005

World Health Organization. (2002). Active ageing: A policy framework. Retrieved from https://www.who.int/ageing/publications/active_ ageing/en/

Yardley, L., Ainsworth, B., Arden-Close, E., \& Muller, I. (2015). The person-based approach to enhancing the acceptability and feasibility of interventions. Pilot and Feasibility Studies, 1(1), 37. doi:10.1186/ s40814-015-0033-z.

Yardley, L., Morrison, L., Bradbury, K., \& Muller, I. (2015). The personbased approach to intervention development: Application to digital health-related behavior change interventions. Journal of Medical Internet Research, 17(1), e30. PubMed ID: 25639757 doi:10.2196/ jmir.4055

Zubala, A., MacGillivray, S., Frost, H., Kroll, T., Skelton, D.A., Gavine, A., ... Morris, J. (2017). Promotion of physical activity interventions for community dwelling older adults: A systematic review of reviews. PLoS One, 12(7), e0180902. doi:10.1371/journal.pone.0180902 